\title{
Quand les robots entrent en classe
}

Thierry Karsenti

Université de Montréal

\section{HRONIQUE • Technologies en éducation}

\section{Introduction}

Aujourd'hui, l'apprentissage du code est devenu essentiel pour les enfants (OCDE, 2015), d'autant plus que cela les rapproche grandement des compétences dites du 21 ${ }^{\mathrm{e}}$ siècle (Ontario Public Service, 2016). Mais pourquoi un tel intérêt pour l'apprentissage du code à l'école? Tout simplement parce que les nouvelles technologies sont de plus en plus présentes aujourd'hui et que les applications et outils numériques du quotidien requièrent aujourd'hui une forte maîtrise et compréhension de l'environnement informatique, des conséquences de telle ou telle manipulation, etc. Il apparaît alors comme évident que l'école aide à ces apprentissages ${ }^{1}$, voire propose un tel enseignement. En utilisant des sites internet, des logiciels, des applications et même des robots! En effet, de nombreux petits robots font aujourd'hui figure d' " outils éducatifs » à des fins d'apprentissage de la programmation à l'école (Misirli et Komis, 2012), mais pas seulement...

\section{Pourquoi les robots?}

L'apprentissage du code, ou de la pensée informatique, n'est pas quelque chose de nouveau, ou de révolutionnaire. En effet, c'est Seymour Papert (1981) qui, avec sa tortue LOGO, a amené les élèves à apprendre à coder, et ce, dès la fin des années 1970. Plus de 40 ans plus tard, l'apprentissage du code revient au premier plan et est parfois même obligatoire. De nombreux outils sont alors intégrés dans les classes, tels que les logiciels de programmation Scratch ou Scratch Jr (Ruf, Mühling et 
Hubwieser, 2014; Sáez-López, Román-González et Vázquez-Cano, 2016), mais aussi le site internet Code.org qui propose une foule de ressources pour les enseignants et les élèves. Ces outils comportent d'ailleurs une foule d'avantages pour les élèves (Moreno-León, Robles et Román-González, 2016). Cependant, pour attirer encore plus les élèves, et donner une autre dimension à l'apprentissage du code, le recours à des robots éducatifs est devenu la nouvelle tendance. En effet, les élèves ne voient plus uniquement le résultat de leur programme sur un écran, comme c'était le cas avec le langage LOGO ou avec des logiciels tels que Scratch, mais bel et bien sur un robot qui va avancer, tourner, reculer, parler, etc. de manière concrète.

\section{Quels sont les principaux robots en éducation?}

Aujourd'hui, on retrouve de nombreux robots dans les salles de classe. Ces robots sont de différents types. On va avoir des robots basés sur le modèle des Lego, et qui vont être montés avant usage, les robots de type Technics, qui sont eux aussi à monter et démonter, les robots qui vont rouler comme une auto, ceux qui vont tourner sur eux-mêmes comme des balles, ceux qui vont sauter, etc. et même certains qui vont marcher et interagir, tels que les robots humanö̈des NAO, même si ces derniers sont plus rares. À des fins de clarté, nous avons souhaité créer une matrice non exhaustive regroupant les principaux robots de sol en éducation et mettant en valeur certaines de leurs caractéristiques, mais aussi les avantages et inconvénients associés à leurs usages en contexte éducatif.

\begin{tabular}{|c|c|c|c|c|}
\hline ROBOT & PUBLIC & CARACTÉRISTIQUES & AVANTAGES & INCONVÉNIENTS \\
\hline SPHERO OLLIE & $\begin{array}{l}\text { Du primaire } \\
\text { au secondaire }\end{array}$ & $\begin{array}{l}\text { Robot ayant une forme } \\
\text { cylindrique et qui peut } \\
\text { réaliser des figures et } \\
\text { des sauts, le tout en le } \\
\text { programmant à l'aide d'une } \\
\text { tablette ou d'un téléphone } \\
\text { intelligent via une connexion } \\
\text { Bluetooth. }\end{array}$ & $\begin{array}{l}\text { - Simple à utiliser } \\
\text { - Adaptable à tout } \\
\text { environnement (sol } \\
\text { goudronné comme tapis } \\
\text { de sol) } \\
\text { - Aspect ludique et futuriste }\end{array}$ & $\begin{array}{l}\text { - Difficile à manipuler en } \\
\text { raison de la réactivité de } \\
\text { l'outil aux commandes } \\
\text { - Limité d'un point de vue } \\
\text { pédagogique }\end{array}$ \\
\hline PRO-BOT & Maternelle & $\begin{array}{l}\text { Se programme à l'aide } \\
\text { de touches fléchées. Un } \\
\text { écran } L C D \text { indique tous les } \\
\text { mouvements programmés. } \\
\text { Chaque consigne correspond } \\
\text { à un déplacement de } \\
25 \mathrm{~cm} \text { ou à une rotation de } \\
25 \text { degrés. }\end{array}$ & $\begin{array}{l}\text { - Excellent robot } \\
\text { d'introduction à la } \\
\text { programmation } \\
\text { - Simple d'utilisation } \\
\text { - De nombreuses } \\
\text { possibilités (avancer, } \\
\text { dessiner, etc.) } \\
\text { - Possibilité de voir le } \\
\text { programme créé sur } \\
\text { l'écran }\end{array}$ & $\begin{array}{l}\text { - Pas de programmation } \\
\text { par boites graphiques } \\
\text { - Design quelque peu } \\
\text { sommaire et trop ciblé } \\
\text { (automobile) }\end{array}$ \\
\hline
\end{tabular}




\begin{tabular}{|c|c|c|c|c|}
\hline$\frac{\frac{\text { PROGRAMMABLE }}{\text { ROBOT MOUSE }}}{\text { ? }}$ & Maternelle & $\begin{array}{l}\text { Outil en forme de souris } \\
\text { que l'on programme en } \\
\text { appuyant sur des flèches } \\
\text { directionnelles. La souris } \\
\text { s'allume, émet des sons et } \\
\text { dispose de } 2 \text { vitesses. }\end{array}$ & $\begin{array}{l}\text { - Destinée à un jeune public } \\
\text { - Programmation ludique et } \\
\text { physique attirant pour les } \\
\text { enfants en maternelle }\end{array}$ & $\begin{array}{l}\text { - Fonctions limitées } \\
\text { en matière de } \\
\text { programmation }\end{array}$ \\
\hline $\begin{array}{l}\text { BLUE-BOT\& } \\
\underline{B E E-B O T}\end{array}$ & Maternelle & $\begin{array}{l}\text { Permet aux utilisateurs } \\
\text { d'appliquer un programme } \\
\text { en appuyant sur le dos du } \\
\text { robot. Programmable en } \\
\text { Bluetooth à partir d'une } \\
\text { tablette tactile. }\end{array}$ & $\begin{array}{l}\text { - Un excellent robot } \\
\text { d'introduction à la } \\
\text { programmation } \\
\text { - Simple d'utilisation } \\
\text { - La possibilité de le } \\
\text { commander via une } \\
\text { tablette }\end{array}$ & $\begin{array}{l}\text { - Fonctions limitées en } \\
\text { programmation }\end{array}$ \\
\hline OZOBOT & $\begin{array}{l}\text { Du primaire } \\
\text { au secondaire }\end{array}$ & $\begin{array}{l}\text { Réagit aux lignes et aux } \\
\text { couleurs qu'il croise sur } \\
\text { son chemin. Possibilité } \\
\text { d'effectuer de nombreuses } \\
\text { activités en utilisant } \\
\text { l'application gratuite Ozobot. }\end{array}$ & $\begin{array}{l}\text { - Permet de travailler la } \\
\text { créativité et la logique } \\
\text { - Utilisable sur une tablette } \\
\text { tactile } \\
\text { - Utilisable avec des } \\
\text { boites graphiques de } \\
\text { programmation }\end{array}$ & $\begin{array}{l}\text { - Petite taille } \\
\text { - Limité pour le travail à } \\
\text { plusieurs }\end{array}$ \\
\hline$\frac{\text { Star Wars Sphero }}{-B B-8}$ & $\begin{array}{l}\text { Du primaire } \\
\text { au secondaire }\end{array}$ & $\begin{array}{l}\text { Inspiré du film Star Wars, il } \\
\text { s'agit d'un robot contrôlable } \\
\text { à l'aide d'une tablette ou } \\
\text { d'un téléphone intelligent. }\end{array}$ & $\begin{array}{l}\text { - Ludique } \\
\text { - Peut-être guidé par } \\
\text { d'autres logiciels de } \\
\text { programmation } \\
\text { - Prise en main aisée }\end{array}$ & $\begin{array}{l}\text { - Fonctionnalités très } \\
\text { basiques } \\
\text { - Outil peut-être plus } \\
\text { commercial qu'éducatif }\end{array}$ \\
\hline
\end{tabular}

\section{Des impacts positifs en éducation}

À la suite de nos observations et entretiens avec les enseignants et les élèves dans les établissements scolaires, nous avons pu mettre en évidence un certain nombre de bénéfices liés aux usages des robots en classe. Ainsi, outre un développement de la motivation majeur pour les élèves, de par la manipulation de tels outils, il a été mis en évidence que ces robots pouvaient aider à l'apprentissage, ou tout du moins à l'initiation à la programmation, et ce, dès le plus jeune âge. En effet, pour une enseignante en maternelle que nous avons interrogée, les « enfants travaillent plus fort » avec de tels outils. Selon une enseignante de $1^{\text {re }}$ année, même les élèves pour qui c'est difficile « restent engagés ». 


\section{CHRONLOULE}

Aussi, il a été observé un développement de la collaboration et de l'entraide entre les élèves dès lors qu'ils manipulaient des robots, comme le confirme une enseignante : " avec l'aide des autres, ils y sont arrivés ». Un autre apprentissage concerne l'orientation spatiale des élèves qui peuvent travailler dans des espaces ouverts, tels que de larges couloirs ou des gymnases, pour expérimenter leurs programmes. Une enseignante, même peu habituée aux usages du numérique en classe, a confié avoir été surprise par la motivation des enfants et le fait que certains d'entre eux soient «fascinés » par l'utilisation de ces outils. On a aussi pu

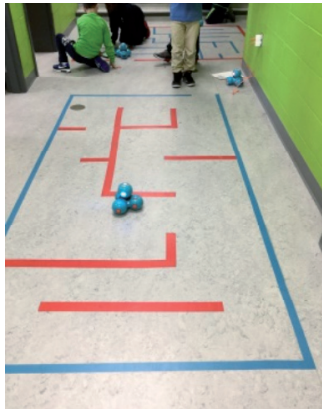
constater une progression de leur " confiance », de leur compréhension de la logique computationnelle, de leurs compétences en résolution de problèmes, de leur "goût d'essayer », de leur plaisir et de leur créativité, qui a une place « immense » dans ces usages.

Toutefois, certains défis subsistent, tels que le fait d'avoir certains élèves qui ne vont pas avancer du tout, alors que d'autres performent dans les activités proposées avec les robots.

\section{Conclusion}

Cette étude a permis de mettre en évidence un certain nombre d'avantages et quelques défis liés à ces usages des robots en contexte scolaire. En dehors d'une hausse forte de la motivation et de l'engagement des élèves, nous avons pu constater une progression de la créativité des élèves, mais aussi de leurs compétences en logique informatique et en résolution de problèmes. Ces avantages majeurs, en particulier en ce qui concerne la motivation et le plaisir pris par les élèves lors de ces activités pédagogiques médiées par des robots, confirment l'intérêt de telles activités pédagogiques.

\section{Note}

1 http://ecolebranchee.com/2016/02/22/les-eleves-de-la-colombie-britannique-apprendront-a-coder/

\section{Références}

Misirli, A. et Komis, V. (2012, 30 mai). Jeux programmables de type Logo à l'école maternelle. Adjectif. Repéré à http://www.adjectif.net/spip/spip.php?article140\&lang=fr

Moreno-León, J., Robles, G. et Román-González, M. (2016). Code to learn: Where does it belong in the K-12 curriculum? Journal of Information Technology Education: Research, 15, 283-303. http://dx.doi.org/10.28945/3521

OCDE. (2015). Connectés pour apprendre? Les élèves et les nouvelles technologies. Principaux résultats. Repéré à https://www.oecd.org/fr/edu/scolaire/Connectes-pour-apprendre-les-eleves-et-les-nouvelles-technologies-principauxresultats.pdf

Ontario Public Service. (2016). Towards defining 21 $1^{\text {st }}$ century competencies for Ontario. Foundation document for discussion. Repéré à http://www.edugains.ca/resources21CL/About21stCentury/21CL 21stCenturyCompetencies.pdf

Papert, S. (1981). Jaillissement de l'esprit : ordinateurs et apprentissage. Paris : Flammarion.

Ruf, A., Mühling, A. et Hubwieser, P. (2014). Scratch vs. Karel: Impact on learning outcomes and motivation. Dans C. Schulte, M. E. Caspersen et J. Gal-Ezer, Proceedings of the $9^{\text {th }}$ workshop in primary and secondary computing education (p. 50-59). New York, NY : ACM. http://dx.doi.org/10.1145/2670757.2670772

\section{Pour citer cet article}

Bugmann, J. et Karsenti, T. (2018). Quand les robots entrent en classe. Formation et Profession, 26(1), 142-145. http://dx.doi.org/10.18162/fp.2018.a141 